\title{
Evaluation of some simplified models for predicting the moisture content of fine, dead fuels.
}

\author{
$\underline{\text { J.J. Sharples }}^{\text {a }}$ and S. Matthews ${ }^{\text {b }}$ \\ ${ }^{a}$ Applied and Industrial Mathematics Research Group, School of Physical, Environmental and Mathematical \\ Sciences, University of New South Wales at the Australian Defence Force Academy, Canberra ACT, \\ Australia \\ Email:j.sharples@adfa.edu.au \\ ${ }^{b}$ CSIRO Ecosystem Sciences, Sydney Australia.
}

\begin{abstract}
Operational prediction of wildfire behaviour requires assessment of the moisture content of fine, dead fuels to within an acceptable degree of accuracy. Ideally the methods of assessment should be simple enough to implement in most operational settings, including those where computational power is a constraining factor. In this paper we compare fine fuel moisture observations relating to two different fuel types with predictions derived from a number of fuel moisture models.

The models considered are: the empirical fuel moisture sub-model of the Western Australian Forest Fire Behaviour Tables ("Red Book"); a process-based model which accounts for heat and moisture fluxes within surface litter; two simplifications of the process-based model; and a very simple model based on a fuel moisture index defined in terms of the difference between air temperature and relative humidity.

The study utilises two sets of fuel moisture data collected, respectively, in Jarrah (Eucalyptus marginata) and Karri (Eucalyptus diversicolor) forests. These two species dominate the forested areas of southwest Western Australia. Specifically, the study considers surface fuel moisture content, which is the moisture content of the top $10 \mathrm{~mm}$ of the litter bed, measured daily at 14:00 hours over the period 13 October $1982-15$ March, 1983. The predictive ability of the various models is evaluated through comparison of the model predictions with observed fuel moisture contents. While changes in the climate over southwest WA since 1975 mean that the data may not be entirely representative of the full range of fine fuel conditions under typical current climatic conditions, the data sets do permit exploration of the various model's performance over the space of relevant meteorological variables.
\end{abstract}

In the present paper the predictive ability of the fuel moisture models is mainly considered in the context of fire management. Of particular interest is the predictive ability of the simpler models at the lower end of the fuel moisture continuum, especially at or below the flammability limit (approximately 25\%). The specific interest in the performance of the simpler models is due to the fact that these models are the most easily implemented in a field setting and hence will be of the most direct use to fire-ground personnel. Model performance is evaluated through consideration of correlation and error statistics, a simple measure of prediction bias and the proportion of model predictions that match observed fuel moisture contents to within a specified tolerance.

It is found that the simple models perform quite well; in fact they outperform the more sophisticated models in a number of the evaluation measures. The results of the study have implications for model engineering and parsimony that are of particular relevance to the application of fine fuel moisture models during bushfire operations.

Keywords: Fuel moisture content, fuel moisture modelling, bushfire, fire management 


\section{INTRODUCTION}

It is well known that the amount of moisture present in fine dead fuels is a key factor affecting fire potential and fire behaviour. For example, the McArthur Forest Fire Danger Index (McArthur 1967) varies approximately as the inverse square of fine fuel moisture content (Matthews 2009). Assessing the moisture content of fine dead fuels to within a reasonable degree of accuracy is therefore an important consideration in fire management practices. Indeed, having access to tools that enable field estimation of fuel moisture content provides fire-ground personnel with knowledge about the expected rate of fire spread, flame height and spotting potential in addition to the general flammability of fuels. The wildfire literature is thus replete with various approaches to modelling fuel moisture content (e.g. Sneeuwjagt and Peet 1985; Wittich 2005; Matthews 2006).

Sharples and McRae (2011) considered a very simple model for estimating the moisture content of eucalypt litter and compared its predictions to those arising from a process-based model (Matthews 2006) and two of its simplifications (Matthews et al. 2010). These studies found that the simple models could provide predictions of fuel moisture content that were comparable to those provided by the more sophisticated process-based model but at a much lower computational cost. The dataset used in these studies, however, only covered a relatively narrow range of conditions and the statistical methods used by Sharples and McRae (2011) were not the most robust. This paper therefore extends previous work by considering a more comprehensive fuel moisture dataset and a broader suite of fuel moisture models.

The main aim of the present paper is to identify conditions for which, in the context of fire management, the simple models perform to a standard that is acceptible for estimation of fuel moisture content, thereby establishing operational thresholds for its use in the field.

\section{MODELS FOR FUEL MOISTURE CONTENT}

\subsection{The WA 'Red Book' moisture content prediction sub-system}

The forest fire behaviour tables for Western Australia (Sneeuwjagt and Peet 1985; Beck 1995), referred to collectively as the 'Red Book', are used to predict fire behaviour in forest types that are common throughout the southwest of WA. This fire behaviour prediction system contains a sub-system used to estimate surface and profile litter moisture contents. For further information on the 'Red Book' fuel moisture sub-model the reader is referred to Beck (1995) who provides a detailed description of the sub-model and derives a set of equations that embodies its content. In the following, FMC predictions arising from the WA 'Red Book' submodel will be denoted by $W A R B$.

\subsection{The process-based model of Matthews (2006)}

Matthews (2006) introduced and described a model which estimates fuel moisture content by accounting for fluxes of energy and water within a litter layer. Heat and water budgets are calculated at five vertically spaced nodes using differential and algebraic equations for the litter temperature, temperature of free liquid water on the litter surface, air temperature, litter moisture content, amount of liquid water on the litter surface and specific humidity. Boundary fluxes are computed from air temperature, wind speed, specific humidity, rainfall rate, solar radiation, thermal radiation, soil temperature and soil moisture (Matthews 2006). Predictions arising from this model will be denoted as $P B M$.

Matthews et al. (2007) also considered PBM estimates using alternate parameter values, which gave the best agreement between observations and model predictions. Such estimates will also be considered in the present study and will be denoted as $P B M^{*}$.

\subsection{The process-based model simplifications}

Matthews et al. (2010) considered two simplifications of the process-based model. Firstly, in the absence of rain, and with a number of simplifying assumptions, the process-based model can be reduced to a single differential equation for the litter moisture content. Predictions arising from this model will be denoted by SEM. Secondly, a table of fuel moisture values is built by applying the process-based model to a wide variety of hypothetical weather conditions. Fuel moisture predictions are then obtained as the table value whose corresponding weather inputs most closely match the observed weather conditions. Predictions arising from this model will be denoted by $T M$. 


\subsection{The fuel moisture index}

The fuel moisture index (FMI) was introduced and discussed by Sharples et al. (2009). It is defined in terms of the difference between air temperature $T\left({ }^{\circ} \mathrm{C}\right)$ and relative humidity $H(\%)$ as:

$$
F M I=10-0.25(T-H),
$$

FMI is a dimensionless index and should not be considered as giving a direct estimate of fuel moisture content. However, Sharples et al. (2009) demonstrated that it was remarkably effective at emulating the predictions of a number of empirical models for fuel moisture content. Moreover, as mentioned above, Sharples and McRae (2011) demonstrated that FMI delivered estimates of fuel moisture content that compared favourably with limited field observations of fuel moisture content.

To obtain predictions of fuel moisture, $F M I$ is multiplied by a scaling factor with units of $\%$, which is obtained empirically. We use the notation $F M I^{*}$ to distinguish the scaled fuel moisture index from the raw fuel moisture index FMI. We use $\alpha$ to denote the scale factor, so that:

$$
F M I^{*}=\alpha F M I \text {. }
$$

The scaling factor $\alpha$ could be derived via a number of methods, but in this study $\alpha$ will be selected so that the mean of the $F M I^{*}$ values, calculated over a suitable calibration subset, equals the mean of the corresponding observed fuel moisture contents.

\section{DATA AND METHODS}

\subsection{Fuel moisture and weather data}

The two sets of fuel moisture observations and their associated weather and boundary flux data described by Matthews et al. (2007) are again employed in the present study. However, given the focus on fire management applications, only the observations recorded at 14:00 hrs, when fuel moisture contents are typically near minimum, are considered. The fuel moisture observations cover the period 13 October $1982-$ 15 March, 1983. In the following $T$ denotes maximum daily temperature $\left({ }^{\circ} \mathrm{C}\right), H$ denotes minimum daily relative humidity (\%), and $m$ denotes observed fuel moisture content at 14:00 hrs (\% of oven-dried weight). The Jarrah dataset comprised 154 observations (1 day missed) with $4 \% \leq m \leq 168 \%$, while the Karri dataset comprised 153 ( 2 days missed) with $7 \% \leq m \leq 121 \%$. Maximum daily temperature ranged between $14^{\circ} \mathrm{C}$ and $42^{\circ} \mathrm{C}$ and minimum daily relative humidity ranged between $14 \%$ and $80 \%$.

Given the focus on fire management applications, the main interest was in model performance for fuel moisture contents below the flammability limit of approximately 25\% (Matthews et al., 2007). Consequently, censored versions of the two datasets were used in the analyses. The censored datasets were comprised of all fuel moisture contents less than or equal to a threshold fuel moisture content, denoted $m_{T}$, along with their associated variables: $T, H$, etc. The maximum threshold fuel moisture content considered was $m_{T}=50 \%$ (approximately twice the flammability limit), while the minimum was $m_{T}=10 \%$.

\subsection{Model performance measures}

The predictive ability of the models over each of the censored datasets is evaluated through calculation of a number of correlation and error statistics. Specifically, the linear correlation coefficient $(\rho)$ between predicted and observed fuel moisture values; the mean absolute error (MAE); the root-mean-square error (RMSE); and a measure of prediction bias $(\varphi)$ are considered. The prediction bias $\varphi$ is defined as the proportion of data for which the predicted fuel moisture value exceeded the observed value. Ideally a bias of $\varphi=0.5$ is desired, though for operational estimation of fuel moisture content in the context of fire management it is perhaps better to have $\varphi$ slightly below 0.5 than slightly above. Under this criterion the model will tend to slightly under-predict fuel moisture content, so that on average fire behaviour will be less dangerous than would be expected based on estimation of fuel moisture content.

Given the absence of independent calibration and validation data, the correlation and error statistics relating to the $F M I^{*}$ model were estimated using $k$-fold cross validation (Geisser, 1995). In $k$-fold cross validation the dataset

$$
D=\left\{\left(T_{i}, H_{i}, m_{i}\right): i=1, \ldots, N\right\}
$$

is randomly split into $k$ disjoint subsets $D_{1}, D_{2}, \ldots, D_{k}$ of approximately equal size. For each $i=1,2, \ldots, k$ the scale factor $\alpha$ is calibrated using the set $D D_{i}$. As mentioned above, $\alpha$ is calibrated so that mean of the fuel 
moisture content values in $D \backslash D_{i}$ equals the mean of the corresponding $F M I^{*}$ values. The subset $D_{i}$ is then used as validation data to allow calculation of the various correlation and error statistics. The $k$ estimates of the correlation and error statistics thus obtained are averaged to provide more robust estimates. In this study we chose $k=5$. The entire 5-fold cross validation process just described was replicated 100 times and the results averaged to obtain the correlation and error statistics reported below.

\section{RESULTS}

The correlation and error statistics corresponding to the various threshold fuel moisture contents for the Jarrah dataset can be seen in Figure 1. The results corresponding to the $P B M$ and $T M$ values displayed similar behaviour to those relating to the $P B M^{*}$ and $S E M$ values, respectively, and so are not shown. Figure 1a indicates that when all $m \leq 50 \%$ were considered the $P B M^{*}$ model yielded the highest correlation, while if $m$ $\leq 30 \%$ the highest correlations were associated with the $F M I^{*}$ model. Figures $1 \mathrm{~b}$ and $1 \mathrm{c}$ indicate that for $m \leq$ $50 \%$ the $F M I^{*}$ model yielded the lowest MAE and RMSE values, while if $m<15 \%$ the $F M I^{*}, P B M^{*}$ and SEM models all yielded similar values of MAE and RMSE. The WARB model had the highest MAE and RMSE values for all values of $m_{T}$. Similarly Figure 1d indicates that the WARB model consistently overpredicted fuel moisture content, with over $80 \%$ of predictions higher than observed values of $m$, regardless of the threshold fuel moisture content. The $P B M^{*}$ tended to slightly under-predict fuel moisture except for low values of $m_{T}$, while $F M I^{*}$ tended to over-predict for $m_{T}>30 \%$ and slightly under-predict for $m_{T}<20 \%$. The $S E M$ model was found to under-predicted fuel moisture content for all values of $m_{T}$.
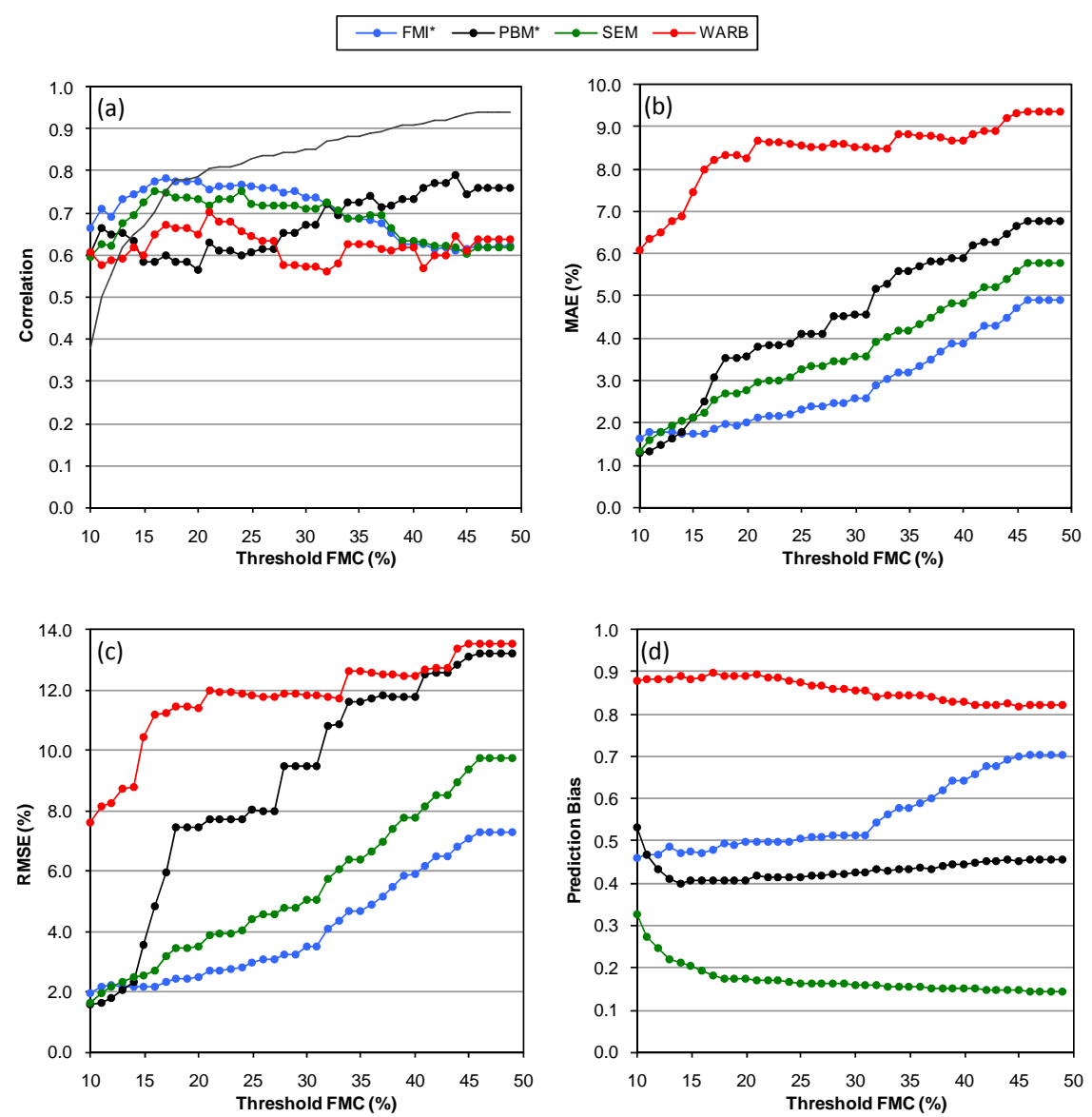

Figure 1. (a) Correlation, (b) mean absolute error, (c) root mean square error, and (d) prediction bias $\varphi$ arising from comparison of $F M I^{*}$ (blue), $P B M^{*}$ (black), SEM (green) and WARB (red) model predictions with observed fuel moisture contents in the Jarrah dataset. The grey line in (a) indicates the proportion of the 154 data points below the threshold FMC.

The analogous results for the Karri dataset are depicted in Figure 2. The $F M I^{*}$ model again yields the highest correlations for $m_{T}<30 \%$ (Figure 2a), and the lowest MAE and RMSE for $m_{T}>20 \%$ (Figure 2b and 2c). For $m_{T} \leq 20 \%$ the SEM model provides the lowest MAE and RMSE values. Surprisingly, the error statistics associated with the $P B M^{*}$ model for $m_{T}>20 \%$ are quite high with MAE $>5 \%$ and RMSE $>12 \%$. As was 

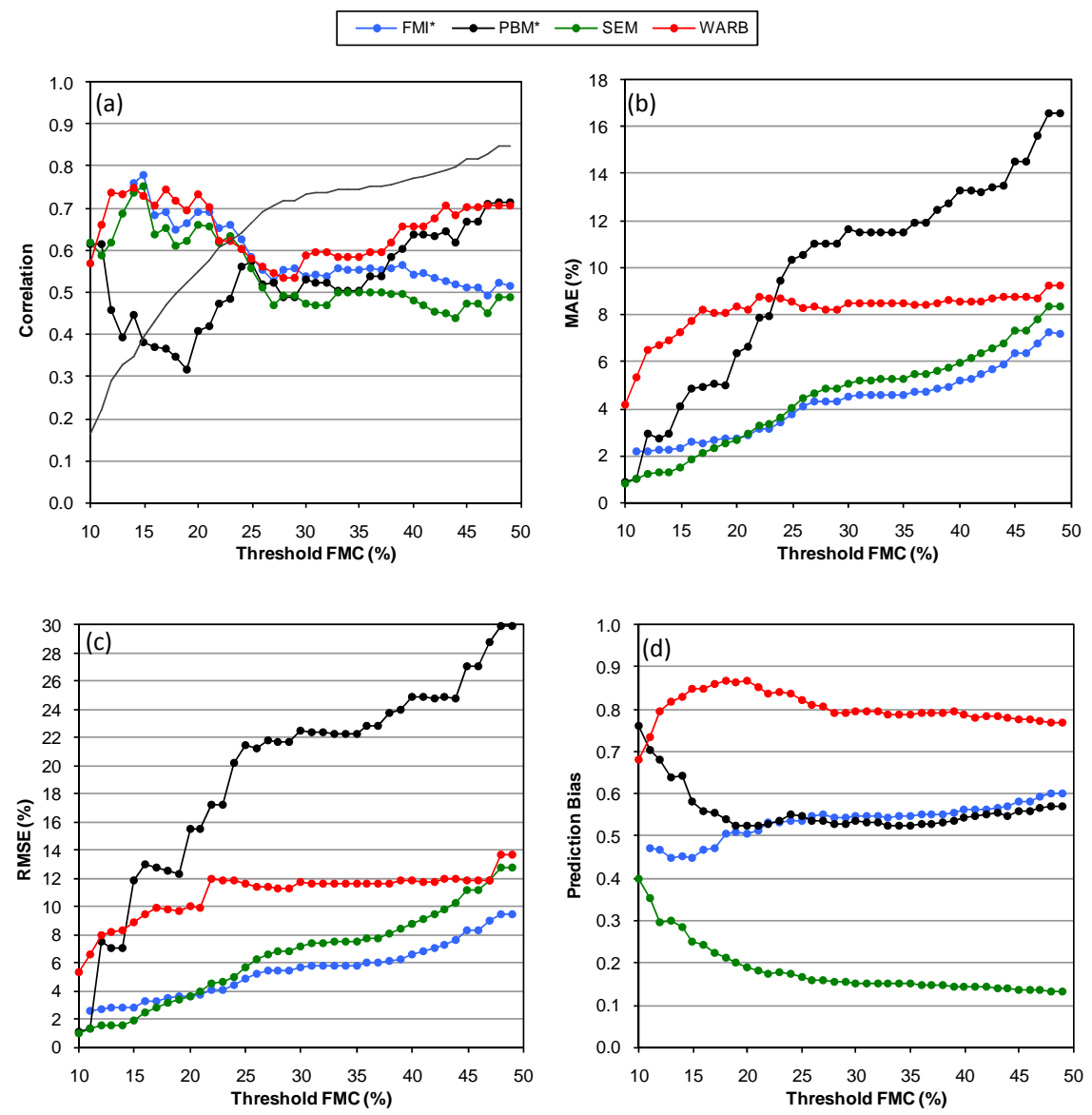

Figure 2. (a) Correlation, (b) mean absolute error, (c) root mean square error, and (d) prediction bias $\varphi$ arising from comparison of $F M I^{*}$ (blue), $P B M^{*}$ (black), SEM (green) and WARB (red) model predictions with observed fuel moisture contents in the Karri dataset. The grey line in panel (a) indicates the proportion of the 153 data points below the threshold FMC.

found in the case of the Jarrah dataset, the $W A R B$ model consistently over-predicted fuel moisture, while the $S E M$ model consistently under-predicted (Figure 2d). The $P B M^{*}$ and $F M I^{*}$ models both over-predicted slightly for $m_{T}>20 \%$. For $m_{T}<20 \%$ the $F M I^{*}$ model slightly under-predicted fuel moisture and the $P B M^{*}$ displayed an increase in the level of over-prediction.

The predictive ability of the various models was also assessed by calculating the proportion of model predictions that matched the fuel moisture observations to within a specified tolerance. Specifically, tolerances of $1 \%, 2 \%$ and $3 \%$ were considered. Figure 3 depicts the results for the Jarrah (top three panels) and Karri (bottom three panels) fuel types. The results for Jarrah litter indicate that the $F M I^{*}, P M B^{*}$ and $S E M$ models provided a similar level of predictive ability for most values of $m_{T}$ and that the $W A R B$ model performed to a much poorer standard in this respect. Similarly for Karri litter the WARB model performed the worst. For the Karri dataset the $F M I^{*}$ performed to a poorer overall standard than the $P M B^{*}$ and $S E M$ models, but yielded a similar proportion of predictions within the specified tolerance in the range $20 \%<m_{T}<$ $25 \%$.

The results discussed above specifically pertaining to fuel moisture content below the flammability limit (assumed 25\%) are summarized in Table 1 . Table 1 contains an additional model denoted $F M I_{a}{ }^{*}$. This model is defined by multiplying the raw $F M I$ values by the average of the two values of the scale factor $\alpha$ obtained using cross-validation on the Jarrah and Karri datasets with $m_{T}=25 \%$. This model, defined by $0.981 F M I$, is included in Table 1 to assess the effects of using a single scale factor for both fuel types. The table entries indicate that when considering the moisture content of Jarrah litter below the flammability limit, the $F M I^{*}$ model performs the best regardless of the statistic used to measure predictive ability. For Karri litter the $F M I^{*}$ performed best in terms of correlation, RMSE and predictive bias; the SEM model performed best in 

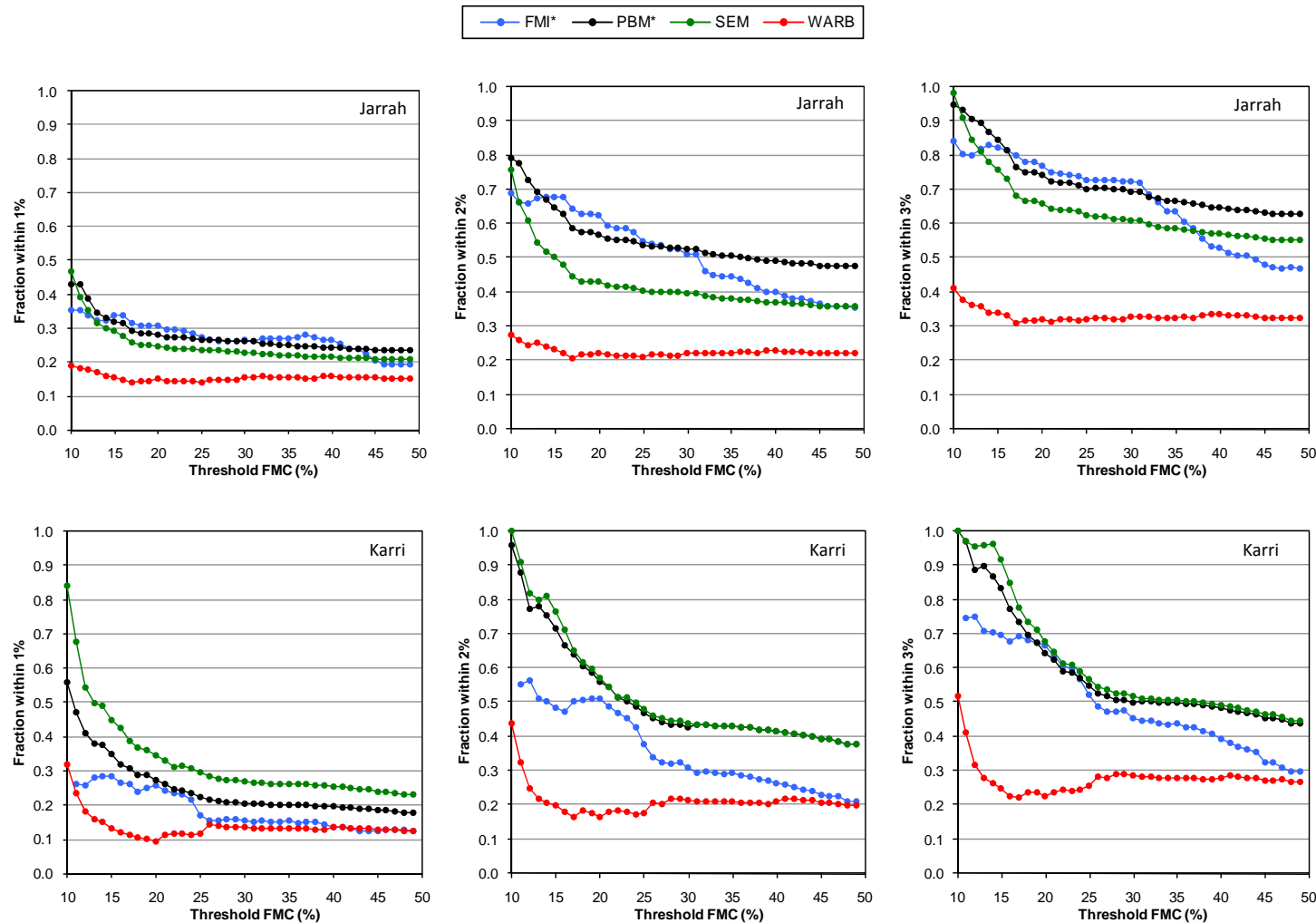

Figure 3. Fraction of the respective model predictions within $1 \%, 2 \%$ and $3 \%$, of the observed fuel moisture contents. The top three panels relate to the Jarrah dataset, the bottom three panels to the Karri dataset.

\begin{tabular}{ccccccccc}
\hline Fuel Type & Model & $\boldsymbol{\rho}$ & MAE (\%) & RMSE (\%) & $\boldsymbol{\varphi}$ & Within 1\% & Within 2\% & Within 3\% \\
\hline \hline Jarrah & $P B M^{*}$ & 0.606 & 4.105 & 8.026 & 0.414 & 0.266 & 0.535 & 0.703 \\
& $S E M$ & 0.722 & 3.252 & 4.414 & 0.164 & 0.234 & 0.403 & 0.625 \\
& $W A R B$ & 0.645 & 8.578 & 11.854 & 0.875 & 0.141 & 0.217 & 0.320 \\
& $F M I^{*}$ & $\mathbf{0 . 7 6 1}$ & $\mathbf{2 . 3 1 5}$ & $\mathbf{2 . 9 7 9}$ & $\mathbf{0 . 5 0 5}$ & $\mathbf{0 . 2 7 2}$ & $\mathbf{0 . 5 4 0}$ & $\mathbf{0 . 7 2 9}$ \\
& $F M I_{a}{ }^{*}$ & $\mathbf{0 . 7 6 1}$ & 2.934 & 3.721 & 0.773 & 0.242 & 0.430 & 0.563 \\
Karri & $P B M^{*}$ & 0.573 & 10.732 & 21.488 & 0.549 & 0.217 & 0.471 & 0.549 \\
& $S E M$ & 0.556 & 4.005 & 5.635 & 0.167 & $\mathbf{0 . 2 8 3}$ & $\mathbf{0 . 4 8 0}$ & 0.569 \\
& $W A R B$ & 0.579 & 8.578 & 11.640 & 0.824 & 0.142 & 0.176 & 0.255 \\
& $F M I^{*}$ & $\mathbf{0 . 5 8 5}$ & 3.754 & $\mathbf{4 . 8 4 2}$ & $\mathbf{0 . 5 3 8}$ & 0.153 & 0.379 & 0.524 \\
& $F M I_{a}{ }^{*}$ & 0.583 & $\mathbf{3 . 5 5 4}$ & 4.898 & 0.353 & 0.235 & 0.412 & $\mathbf{0 . 5 7 8}$ \\
\hline
\end{tabular}

Table 1. Statistics arising from analyses of the various model predictions corresponding to observed fuel moisture contents $m \leq 25 \%$. Note that the model denoted $F M I_{a}{ }^{*}$ corresponds to the $F M I$ with a scale factor of $\alpha=0.981$, which is the average of the values of $\alpha$ derived using cross-validation on the Jarrah and Karri datasets with $m_{T}=25 \%$. Entries in bold identify the model exhibiting the best performance in terms of the relevant statistic.

terms of predicting to within $1 \%$ or $2 \%$ of the observed values; while the $F M I_{a}{ }^{*}$ model performed best in terms of MAE and predicting to within $3 \%$ of the observed values.

\section{DISCUSSION AND CONCLUSIONS}

The performance of a number of simple models for the moisture content of litter was evaluated by comparison with predictions from a process-based model and with observations of fuel moisture content in two WA forest types. Overall, the simple indices were found to perform the best, with the most simple FMI* 
model providing the best results overall. The $P B M$ and $P B M^{*}$ models tended to over-predict fuel moisture content to a significant degree in many instances thus resulting in large values for the error statistics at all but the very lowest threshold fuel moisture contents considered. However, even under these conditions the error statistics for the $P B M$ and $P B M^{*}$ models were not significantly different to those for the simpler $S E M$ and $F M I^{*}$ models. The $W A R B$ model performed the worst overall with consistently large prediction errors and large positive prediction bias.

The $F M I^{*}, S E M$ and TM models tend to saturate as fuel moisture increases and so there is less capacity for the huge over-predictions seen with the $P B M$ and $W A R B$ models. This implies that these simpler models would perform poorly in a contingency table ('burn' or 'no burn') analysis. However, given that the interest is in evaluating the utility of the simpler models in the context of fire management this aspect of their performance does not constitute a significant shortcoming. In fact the results suggest that in the context of fire management, where the conditions encountered are typically at the drier end of the fuel moisture continuum, the $F M I^{*}$ model provides the best guidance overall.

The $F M I^{*}$ model does require calibration against historical fuel moisture data before it can be used to provide direct estimates of fuel moisture content but this could perhaps be accommodated by an appropriate survey of fuel moisture in a particular area where use of the index is proposed and the production of simple conversion tables or graphs that incorporate the appropriate value of the scale factor $\alpha$ for each dominant fuel type. The values of $\alpha$ varied only a small amount with $m_{T}$ for each of the data sets: for the Jarrah data set $\alpha$ varied linearly with $m_{T}$, from $\alpha=1.007$ at $m_{T}=50 \%$ to $\alpha=0.757$ at $m_{T}=10 \%$; for the Karri data set $\alpha$ varied approximately linearly with $m_{T}$, from $\alpha=1.404$ at $m_{T}=50 \%$ to $\alpha=1.076$ at $m_{T}=10 \%$. The reason for the different values of the scale factor obtained in the two forest types is unclear (different values were also obtained by Sharples and McRae (2011)), but presumably is related to the way the different fuel types respond to changes in environmental conditions. This will be investigated more thoroughly in future work.

The fact that very simple models for fuel moisture content can consistently outperform more complex models again raises the point about parsimony of fuel moisture models. This point is particularly relevant for models that are intended to provide operational guidance on a fire-ground where computational resources are limited.

\section{ACKNOWLEDGMENTS}

The authors are grateful to L. McCaw for provision of the fuel moisture data sets and the WA 'Red Book' sub-model predictions.

\section{REFERENCES}

Beck, J.A. (1995) Equations for the Forest Fire Behaviour Tables for Western Australia. CALMScience, 1: 325-348.

Geisser, S. (1995) Predictive Inference: An Introduction. Chapman and Hall, New York.

Matthews, S. (2006) A process-based model of fine fuel moisture. International Journal of Wildland Fire, 15: $155-168$

Matthews, S., McCaw, W.L., Neal, J. and Smith, R. (2007) Testing a process-based fine fuel moisture model in two forest types. Canadian Journal of Forest Research 37: 23-35.

Matthews, S. (2009) A comparison of fire danger rating systems for use in forests. Australian Meteorological and Oceanographic Journal, 58: 41-48.

Matthews, S., Gould, J. and McCaw, L. (2010) Simple models for predicting dead fuel moisture in eucalypt forests. International Journal of Wildland Fire, 19: 459-467.

McArthur, A.G. (1967) Fire behaviour in eucalypt forests. Commonwealth of Australia Forestry and Timber Bureau Leaflet 107. 36pp.

Sharples, J.J., McRae, R.H.D., Weber, R.O. and Gill, A.M. (2009) A simple index for assessing fuel moisture content. Environmental Modelling and Software, 24: 637-646.

Sharples, J.J. and McRae, R.H.D. (2011) Evaluation of a very simple model for predicting the moisture content of eucalypt litter. International Journal of Wildland Fire, in press.

Sneeuwjagt, R.J. and Peet, G.B. (1985) Forest Fire Behaviour Tables for Western Australia. $3^{\text {rd }}$ Edition. Department of Conservation and Land Management: Perth, WA.

Wittich, K-P. (2005) A single-layer litter-moisture model for estimating forest fire danger. Meteorologische Zeitschrift, 14: 157-164. 